\title{
Crescimento e qualidade de mudas de Timbó e Dedaleiro cultivadas em solo contaminado por cobre
}

\author{
Rodrigo F. da Silva ${ }^{1}$, Fábio L. F. Saidelles ${ }^{2}$, Pedro D. C. Kemerich ${ }^{1}$, \\ Ricardo B. Steffen ${ }^{3}$, Alexandre Swarowsky ${ }^{4}$ \& Alessandro S. da Silva ${ }^{5}$
}

\begin{abstract}
RESUMO
As atividades de mineração e de viticultura do Rio Grande do Sul têm contribuído para a contaminação do solo por cobre. Este trabalho objetivou avaliar o efeito de doses de cobre no crescimento e na qualidade de mudas de timbó (Ateleia glazioviana) e dedaleiro (Lafoensia pacarı). O delineamento foi inteiramente casualizado em arranjo fatorial $(2 \times 4)$, formado de duas espécies florestais e quatro doses de cobre $(0,64$, 128 e $192 \mathrm{mg} \mathrm{kg}^{-1}$ de solo) com cinco repetições. Avaliaram-se altura da planta, diâmetro do colo, peso da matéria seca radicular e da parte aérea, comprimento e área superficial específica radicular e os seguintes índices de qualidade de mudas: relação entre altura da parte aérea e diâmetro do coleto; altura da parte aérea e peso da matéria seca da parte aérea e o índice de qualidade de Dickson, em que os resultados revelaram que as doses de cobre testada reduziram a qualidade de mudas do timbó enquanto o dedaleiro apresentou melhor qualidade com $128 \mathrm{mg} \mathrm{kg}^{-1}$ de $\mathrm{Cu}$. O cobre influenciou os parâmetros de crescimento das mudas de timbó porém aumentou significativamente o comprimento e a área superficial específica do dedaleiro até $128 \mathrm{mg} \mathrm{kg}^{-1}$ de $\mathrm{Cu}$. Mudas de dedaleiro são mais tolerantes à contaminação do solo que as de timbó e apresentam melhor qualidade em doses mais elevadas de cobre.
\end{abstract}

Palavras-chave: espécies florestais, metal pesado, tolerância

\section{Growth and quality of Ateleia glazioviana and Lafoensia pacari seedlings cultivated in copper contaminated soil}

\begin{abstract}
The mining and viticulture activities in the State of Rio Grande do Sul, Brazil have contributed to soil contamination by copper. The aim of this study was to evaluate the effect of copper doses on growth and quality of Ateleia glazioviana and Lafoensia pacari seedlings. The experimental design was completely randomized in a factorial scheme $(2 \times 4)$ with five replications. The experiment involved two forest species and three copper doses $\left(64,128\right.$ and $\left.192 \mathrm{mg} \mathrm{kg}^{-1}\right)$. The plant height, stem diameter, root and shoot dry-mass, length and specific superficial area of roots, as well as seedlings quality index: relationship between shoot height and stem diameter, shoot height and dry-mass of shoots were evaluated. In addition, Dickson's quality index was estimated. The results showed that the tested copper doses reduce the quality of seedlings of Ateleia glazioviana; however, the Lafoensia pacari quality was better with Cu dose of $128 \mathrm{mg} \mathrm{kg}^{-1}$. Dose of copper influenced the growth of Ateleia glazioviana seedlings, but increased significantly the length and specific surface area of the Lafoensia pacari up to Cu dose of $128 \mathrm{mg} \mathrm{kg}^{-1}$. Lafoensia pacari seedlings are more tolerant to soil contamination than Ateleia glazioviana and display better quality of seedlings at high copper doses.
\end{abstract}

Key words: forestry species, heavy metal, tolerance

\footnotetext{
CESNORS/UFSM, Linha 7 de Setembro s/n, C. P. n. 54, CEP 98400-000, Frederico Westphalen, RS. E-mail: rodrigosilva@smail.ufsm.br; eng.kemerich@yahoo.com.br

${ }^{2}$ Estação de Pesquisas Florestais do Rio Grande do Sul - FEPAGO Florestas. Boca do Monte, $7^{\circ}$ Distrito, C.P. 346. CEP 97001-970, Santa Maria, RS. E-mail: ffleig@yahoo.com.br

PPGCS/UFSM, Avenida Roraima n.1000, Bairro Camobi, CEP 97015-900, Santa Maria, RS. E-mail: bemfica_steffen@yahoo.com.br

${ }^{4}$ Centro Universitário Franciscano - UNIFRA. Rua dos Andradas, 1614, CEP 97000-000, Santa Maria, RS. E-mail: aleswar@gmail.com

5 FUNASA, SAUS Quadra 4, Bloco N, CEP 70070-040, Brasília, DF. E-mail: alessandromardini@gmail.com
} 


\section{INTRODUÇÃO}

A contaminação de solo por cobre tem-se tornado evidente em várias regiões do Brasil, devido às atividades de mineração e à produção de videiras em decorrência da aplicação de fungicidas à base de cobre (Chaignon \& Hinsinger, 2003; Nachtigall et al., 2007). Em áreas contaminadas por metais ocorrem danos ao meio ambiente, pela ação desses elementos sobre a vegetação. Deste modo, a escolha adequada de espécies utilizadas em processos de revegetação é muito importante, devido à necessidade de rápida adaptação aos fatores limitantes de crescimento da planta, sobretudo quando se trata de solos contaminados por metais (Pralon \& Martins, 2001).

O cobre é um elemento essencial para produtividade das plantas e participa do metabolismo de carboidratos, do nitrogênio, da síntese de lignina e clorofila (Dechen \& Nachtigall, 2006). Entretanto, quando atinge elevadas concentrações no solo, variando de $40 \mathrm{mg} \mathrm{kg}^{-1}$ para solo arenoso e $100 \mathrm{mg} \mathrm{kg}^{-1}$ para solo argiloso (Accioly \& Siqueira, 2000), pode ocorrer necrose das folhas, desfolhamento precoce e redução no crescimento do sistema radicular (Bellion et al., 2006). Porém algumas plantas podem tolerar níveis elevados desse elemento podendo acumular concentração superior a $1000 \mathrm{mg} \mathrm{kg}^{-1}$ de massa seca, por meio de mecanismos bioquímicos (Accioly \& Siqueira, 2000). Neste caso, tornam-se convenientes a elaboração de estudos relacionados às espécies florestais arbóreas nativas do Brasil e sua tolerância à toxidez do cobre.

No que se refere às espécies arbóreas, o timbó (Ateleia glazioviana Bail.) é uma árvore de ocorrência natural nas bacias dos Rios Paraguai e Uruguai compondo a vegetação secundária da Floresta Estacional Decidual, razão por que é recomendada para recuperação de áreas degradadas, por apresentar elevada rusticidade (Backes \& Irgang, 2002). O dedaleiro (Lafoensia pacari St. Hil.) é uma espécie nativa encontrada nas florestas de altitude de Minas Gerais até Santa Catarina, sendo indicada para o paisagismo urbano, extração de corantes, construção civil e marcenaria, tal como, também, para reflorestamentos mistos destinados à recomposição de áreas degradadas (Backes $\&$ Irgang, 2002). Contudo, pouco se sabe a respeito do comportamento de timbó e dedaleiro a elevadas doses de cobre no solo.

A avaliação da qualidade das mudas de plantas arbóreas ainda no viveiro, por meio de índices que expressam relações entre os parâmetros de crescimento, pode ser uma ferramenta para identificar seu adequado desenvolvimento e se as mudas estão com o máximo potencial para sobrevivência após o transplante para o campo. A relação altura/peso da matéria seca da parte aérea pode indicar o estado de lignificação da muda, sendo importante para sobrevivência pós-plantio no campo (Gomes et al., 2003), enquanto a relação altura/diâmetro se refere a estiolamento da muda (Souza et al., 2006). Já o índice de qualidade de Dickson leva em consideração a produção da matéria seca da parte aérea, das raízes e total, a altura e o diâmetro de coleto das plantas (Dickson et al., 1960) e tem sido o mais utilizado para avaliar a qualidade de mudas.

Algumas espécies arbóreas nativas são potencialmente aptas para plantações, concorrendo para a diversificação de oferta de matéria-prima visando fins mais nobres, como serraria, laminação e indústria moveleira (Souza et al., 2005). Neste caso, a seleção prévia em substrato contaminado pode fornecer informações adequadas sobre a capacidade de sobrevivência da muda pós-plantio para o campo. Assim, o trabalho teve o objetivo de avaliar o efeito de doses de cobre sobre o crescimento e qualidade de mudas de timbó e dedaleiro.

\section{Material e MÉTODOS}

O experimento foi conduzido em casa de vegetação da Estação de Pesquisas Florestais da Fundação Estadual de Pesquisa Agropecuária (FEPAGRO - Floresta) em Santa Maria, RS. O solo utilizado para compor as unidades experimentais foi um Argissolo Vermelho-amarelo (EMBRAPA, 2006). A análise química do solo revelou $\mathrm{pH}_{\text {agua }}: 5,0 ; \mathrm{Ca}+\mathrm{Mg}: 8,4 \mathrm{cmol}_{\mathrm{c}} \mathrm{dm}^{-1}$, $\mathrm{H}+\mathrm{Al}: 5,5 \mathrm{cmol} \mathrm{dm}^{-1}, \mathrm{P}: 20,8 \mathrm{mg} \mathrm{kg}^{-1}, \mathrm{~K}: 212 \mathrm{mg} \mathrm{kg}^{-1}$, matéria orgânica: $2,5 \mathrm{~g} \mathrm{~kg}^{-1}$, argila: $21 \mathrm{~g} \mathrm{~kg}^{-1}$ e cobre extraído por $\mathrm{HCl}$ : $0,8 \mathrm{mg} \mathrm{kg}^{-1}$. Para elevar o $\mathrm{pH}$ para 5,5, foi adicionada uma mistura de carbonato de cálcio $\left(\mathrm{CaCO}_{3}\right)$ e carbonato de magnésio $\left(\mathrm{MgCO}_{3}\right)$ na relação molar 3:1 aguardando-se 53 dias até a estabilização do $\mathrm{pH}$; enfim aplicou-se, no plantio, o equivalente a $30 \mathrm{~kg} \mathrm{ha}^{-1} \mathrm{de} \mathrm{N}$ na forma de ureia.

As espécies arbóreas nativas utilizadas foram timbó (Ateleia glazioviana Bail.) e dedaleiro (Lafoensia pacari St. Hil.). As mudas foram formadas em viveiro, em bandeja de isopor, tendose como substrato areia lavada e, quando apresentaram um par de folhas definitivas, foram transplantadas para os vasos de cultivo com $500 \mathrm{~g}$ de solo.

O delineamento experimental foi inteiramente casualizado num arranjo fatorial ( $2 \times 4)$, qualitativo em A (duas espécies florestais) e quantitativo em $\mathrm{D}$ (quatro doses de cobre: 0 - nível natural do solo, 64, 128 e, $192 \mathrm{mg} \mathrm{kg}^{-1}$ de solo), com cinco repetições. $\mathrm{O}$ cobre foi aplicado como solução de sulfato de cobre $\left(\mathrm{CuSO}_{4} 5 \mathrm{H}_{2} \mathrm{O}\right)$ de forma suplementar. $\mathrm{O}$ experimento foi conduzido durante seis meses, mantendo o solo com aproximadamente $80 \%$ da capacidade de campo.

Os parâmetros analisados foram: altura de planta (AP), diâmetro do colo (DC), peso da matéria seca radicular (PMSR) e da parte aérea (PMSA), comprimento radicular (CR) e área superficial específica radicular (ASE) conforme Tennant (1975). A altura de parte aérea $(\mathrm{cm})$ foi medida com régua graduada do colo da planta até o meristema apical. O diâmetro do colo da muda foi tomado com paquímetro digital, com precisão de 0,01 $\mathrm{mm}$. Para quantificação da matéria seca o sistema radicular foi separado da parte aérea e ambos foram secados em estufa de circulação forçada a $60{ }^{\circ} \mathrm{C}$, até peso constante, pesados em balança com precisão de $0,01 \mathrm{~g}$.

Foram calculados, com base nos parâmetros morfológicos avaliados, os índices de qualidade de mudas: relação entre altura da parte aérea e diâmetro do coleto (H/DC); altura da parte aérea e peso de matéria seca da parte aérea (H/PMSPA) e o Índice de Qualidade de Dickson (IQD) (Dickson et al., 1960): $\mathrm{IQD}=\operatorname{PMST}(\mathrm{g}) /[\mathrm{AP}(\mathrm{cm}) / \mathrm{DC}(\mathrm{mm})]+[\operatorname{PMSPA}(\mathrm{g}) / \operatorname{PMSR}(\mathrm{g})]$, sendo PMST o peso de matéria seca total obtido pela soma do PMSPA e o PMSR.

Os resultados foram submetidos a análise de variância e, quando da significância dos efeitos apontados pela análise, as 
médias foram comparadas pelo teste de Tukey e ajustadas equações de regressão pelo programa SISVAR (Ferreira, 2006), com base nos níveis de significância maiores que 95\% ( $\mathrm{p} \leq$ 0,05). Para determinação da influência das concentrações de cobre no crescimento das mudas de timbó e dedaleiro, também se realizou análise dos componentes principais pelo software CANOCO Versão 4.0 (Ter-Braak \& Smilauer, 1998).

\section{RESULTADOS E DISCUSSÃO}

Não houve diferença significativa na altura, diâmetro nem no peso da matéria seca da parte aérea das mudas de dedaleiro submetidas às doses de cobre (Tabela 1). Entretanto, observase diferença significativa $(\mathrm{p} \leq 0,05)$ nos parâmetros radiculares, como peso da massa seca radicular, comprimento e área superficial específica radicular que apresentaram aumento até a dose de $128 \mathrm{mg} \mathrm{Cu} \mathrm{kg}^{-1}$ de solo, sendo posteriormente reduzidos. Embora o cobre seja um elemento essencial para as plantas, em virtude de participar do metabolismo de carboidratos e do nitrogênio pode, quando em altas concentrações, causar efeito tóxico, reduzindo o crescimento vegetal (Dechen \& Nachtigall, 2006). Assim, as mudas de dedaleiro parecem aproveitar o cobre até a dose de $128 \mathrm{mg} \mathrm{kg}^{-1}$ de solo, manifestando toxidez em seu sistema radicular, na dose de $192 \mathrm{mg} \mathrm{kg}^{-1}$ de cobre. Apesar disto, as mudas de timbó não manifestaram diferença significativa nos parâmetros de crescimento: altura, diâmetro e massa seca da parte aérea nem na massa seca radicular, comprimento e área superficial específica radicular (Tabela 1). Marques et al. (2000) não verificaram resposta a metais no solo pela espécie Cedrela fissilis, outra planta arbórea considerada nativa da Região Sul do Brasil, chegando a ser estimulada pelos níveis de contaminação. Neste sentido, as mudas de timbó se mantiveram indiferentes às doses de cobre aplicadas ao solo, neste trabalho.

Mudas de dedaleiro apresentaram maiores valores que as de timbó na altura, em todas as doses de cobre testadas, no peso da massa seca aérea, peso da massa seca radicular, comprimento e área superficial específica radicular com aplicação de $128 \mathrm{mg} \mathrm{Cu} \mathrm{kg}^{-1}$ de solo (Tabela 2). Aárea superficial específica radicular é um parâmetro de grande importância para obtenção de sistemas radiculares com maior capacidade de absorção de nutrientes. Isto significa que para um mesmo peso de raízes aquela que apresentar maior área superficial específica, irá possuir maior quantidade de raízes finas (Tennant, 1975) e, consequentemente, terá maior capacidade de absorção de nutrientes. O maior comprimento e área superficial específica radicular das mudas de dedaleiro em relação às de timbó podem, em doses elevadas de cobre, contribuir para o melhor estabelecimento dessa espécie em solo contaminado (Tabela 2, Figura 1).

Com a análise multivariada dos dados obtidos neste trabalho (Figura 1), observou-se variação quanto à eficiência dos tratamentos expressa por dois eixos para as espécies timbó (Figura 1A) e dedaleiro (Figura 1B). Para a espécie timbó as variáveis são separadas em dois grupos de resposta, o primeiro englobando as concentrações de 0, 64 e $128 \mathrm{mg} \mathrm{Cu} \mathrm{kg}^{-1}$ de solo, as quais estão influenciando a produção de massa seca, tanto radicular como aérea, altura da planta, diâmetro do colo, volume radicular e área superficial específica radicular; o segundo grupo engloba a concentração de $192 \mathrm{mg} \mathrm{Cu} \mathrm{kg}^{-1} \mathrm{de}$ solo na qual o crescimento vegetal foi limitado (Figura 1A). A

Tabela 1. Altura, diâmetro, peso da massa seca aérea (PMSA), peso da massa seca radicular (PMSR), comprimento (CR) e área superficial específica radicular (ASE) de mudas de timbó e dedaleiro submetidas a doses de cobre

\begin{tabular}{|c|c|c|c|c|c|c|}
\hline Dose $\left(\mathrm{mg} \mathrm{kg}^{-1}\right)$ & Timbó & Dedaleiro & Timbó & Dedaleiro & Timbó & Dedaleiro \\
\hline & \multicolumn{2}{|c|}{ Altura (cm) } & \multicolumn{2}{|c|}{ Diâmetro (mm) } & \multicolumn{2}{|c|}{ PMSA (g) } \\
\hline 0 & $8,84 \mathrm{Ba}^{*}$ & $37,04 \mathrm{Aa}$ & $4,44 \mathrm{Aa}$ & $3,76 \mathrm{Aa}$ & $0,62 \mathrm{Ba}$ & $2,26 \mathrm{Aa}$ \\
\hline 64 & 9,12 Ba & $29,10 \mathrm{Aa}$ & 4,27 $\mathrm{Aa}$ & 3,61 Aa & $0,56 \mathrm{Ba}$ & $1,96 \mathrm{Aa}$ \\
\hline 128 & $9,56 \mathrm{Ba}$ & $29,86 \mathrm{Aa}$ & 3,58 Aa & 3,74 $\mathrm{Aa}$ & $0,53 \mathrm{Ba}$ & 2,20 Aa \\
\hline 192 & $9,08 \mathrm{Aa}$ & $19,68 \mathrm{Aa}$ & $3,74 \mathrm{Aa}$ & $2,85 \mathrm{Aa}$ & $0,44 \mathrm{Aa}$ & $1,18 \mathrm{Aa}$ \\
\hline \multirow[t]{2}{*}{ CV (\%) } & \multicolumn{2}{|c|}{30,4} & \multicolumn{2}{|c|}{6,6} & \multicolumn{2}{|c|}{20,0} \\
\hline & \multicolumn{2}{|c|}{ PMSR (g) } & \multicolumn{2}{|c|}{ CR (cm) } & \multicolumn{2}{|c|}{ ASE $\left(\mathrm{cm}^{2}\right)$} \\
\hline 0 & $0,44 \mathrm{Aa}$ & $0,49 \mathrm{Aab}$ & $245,90 \mathrm{Aa}$ & $186,0 \mathrm{Ab}$ & $51,80 \mathrm{Aa}$ & $47,2 \mathrm{Ab}$ \\
\hline 64 & $0,26 \mathrm{Aa}$ & $0,41 \mathrm{Aab}$ & $210,80 \mathrm{Aa}$ & 255,3 Aab & $49,20 \mathrm{Aa}$ & $62,8 \mathrm{Aab}$ \\
\hline 128 & $0,22 \mathrm{Ba}$ & $0,70 \mathrm{Aa}$ & $235,00 \mathrm{Ba}$ & $390,3 \mathrm{Aa}$ & $54,20 \mathrm{Ba}$ & $88,7 \mathrm{Aa}$ \\
\hline 192 & $0,20 \mathrm{Aa}$ & $0,24 \mathrm{Ab}$ & $144,90 \mathrm{Aa}$ & $154,3 \mathrm{Ab}$ & $33,50 \mathrm{Aa}$ & $32,9 \mathrm{Ac}$ \\
\hline CV (\%) & \multicolumn{2}{|c|}{6,8} & \multicolumn{2}{|c|}{17,34} & \multicolumn{2}{|c|}{15,61} \\
\hline
\end{tabular}

Tabela 2. Relação entre altura da parte aérea e peso da matéria seca da parte aérea (H/MSPA), altura e diâmetro (H/D) e índice de qualidade de Dickson (IQD) de mudas de timbó e dedaleiro submetidas a doses de cobre

\begin{tabular}{|c|c|c|c|c|c|c|}
\hline \multirow{2}{*}{$\begin{array}{c}\text { Dose } \\
\mathrm{mg} \mathrm{kg}^{-1}\end{array}$} & \multicolumn{2}{|c|}{ H/PMSA } & \multicolumn{2}{|c|}{$H / D$} & \multicolumn{2}{|c|}{ IQD } \\
\hline & Timbó & Dedaleiro & Timbó & Dedaleiro & Timbó & Dedaleiro \\
\hline 0 & $14,54 \mathrm{Aa}^{*}$ & $16,70 \mathrm{Aa}$ & $2,00 \mathrm{Ba}$ & $9,85 \mathrm{Aa}$ & $0,31 \mathrm{Aa}$ & 0,19 Bab \\
\hline 64 & $16,42 \mathrm{Aa}$ & $16,42 \mathrm{Aa}$ & $2,14 \mathrm{Ba}$ & $8,31 \mathrm{Aa}$ & 0,19 Aab & $0,18 \mathrm{Aab}$ \\
\hline 128 & $18,07 \mathrm{Aa}$ & $14,36 \mathrm{Aa}$ & $2,68 \mathrm{Ba}$ & 7,98 $\mathrm{Aa}$ & $0,15 \mathrm{Bb}$ & $0,26 \mathrm{Aa}$ \\
\hline 192 & $21,12 \mathrm{Aa}$ & $20,08 \mathrm{Aa}$ & $2,43 \mathrm{Ba}$ & $6,97 \mathrm{Aa}$ & $0,14 \mathrm{Ab}$ & $0,12 \mathrm{Ab}$ \\
\hline CV (\%) & \multicolumn{2}{|c|}{14,25} & \multicolumn{2}{|c|}{32,37} & \multicolumn{2}{|c|}{4,97} \\
\hline
\end{tabular}

* Médias seguidas da mesma letra minúscula na coluna e maiúscula na linha, dentro de cada variável, não diferem entre si pelo teste de Tukey a 5\% de probabilidade ( $\mathrm{p}<0,05)$ 


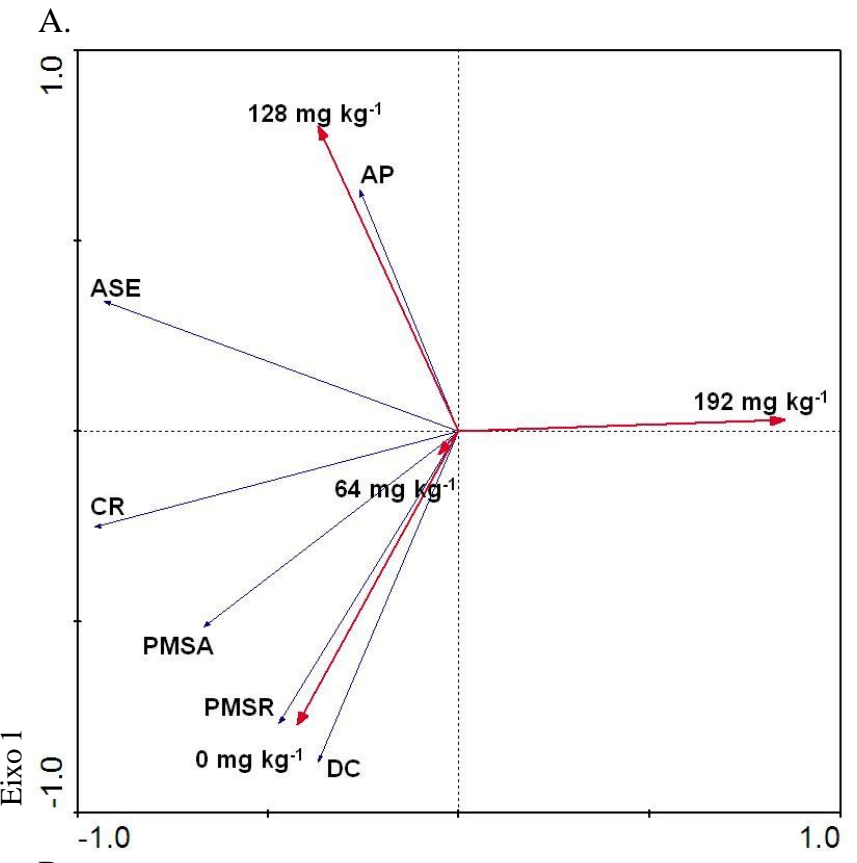

B.

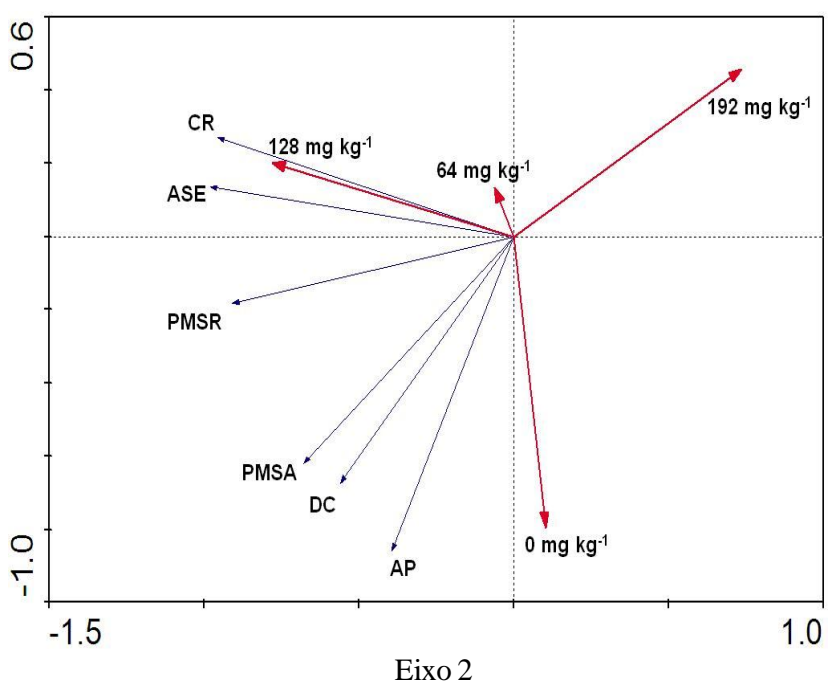

(AP) altura da planta, (CR) comprimento radicular, (DC) diâmetro do colo (ASE) área superficial especíica radicular, (PMSA) peso da matéria seca aérea e (PMSR) peso da matéria seca radicular

Figura 1. Representação gráfica da análise de componentes principais $(\mathrm{PCA})$ relacionando as dimensões 1 e 2 referentes ao desenvolvimento das plantas de timbó (A) e dedaleiro (B) submetidas a diferentes concentrações de cobre

análise dos componentes principais (Figura 1A) mostrou-se o método mais sensível para determinação da influência das doses de cobre sobre os parâmetros de crescimento de mudas de timbó em relação à análise convencional (Tabela 1).

Para o dedaleiro, tal como para o timbó, as variáveis foram separadas em dois grupos de resposta (Figura 1B). Observa-se que o crescimento radicular foi maior em concentrações próximas a $128 \mathrm{mg} \mathrm{kg}^{-1}$. Conforme Almeida et al. (2005), a biomassa radicular irá proporcionar melhor desenvolvimento das plantas quando transferidas para campo em estágio de muda, aumentando a probabilidade de sobrevivência, influenciada pela maior capacidade de sustentação e maior área de absorção de água e nutrientes já a parte aérea do dedalheiro apresentou maior crescimento em menores concentrações de cobre. As concentrações de 0 e $192 \mathrm{mg} \mathrm{Cu} \mathrm{kg}^{-1}$ de solo corresponderam às que apresentaram menor crescimento vegetal (Figura 1B), confirmando os resultados da análise estatística convencional para as mudas de dedaleiro (Tabela 1).

Não houve diferença significativa na relação H/PMSA para as mudas de timbó e dedaleiro cultivadas em solo acrescido de cobre (Tabela 2) mas, conforme Brissete \& Barnett (1991) os valores apresentados por essas plantas estão acima do considerado ideal para este índice, que é de aproximadamente 2,00, sem definição de espécie. Gomes et al. (2003) relataram que, quanto menor a relação H/PMSA mais lignificada estará a muda e maior será sua capacidade de sobrevivência no campo, caso em que a dose de $128 \mathrm{mg} \mathrm{Cu} \mathrm{kg}^{-1}$ de solo parece favorecer as mudas de dedaleiro por proporcionar valor da relação $\mathrm{H} /$ PMSA mais próximo do ideal, em relação às demais doses testadas.

Para a relação H/D também não se observa diferença significativa nas mudas de timbó e de dedaleiro submetidas à aplicação das doses de cobre (Tabela 2). Contudo, os valores apresentados pelo timbó em todas as doses de cobre estão inferiores aos da faixa considerada adequada para essa relação, isto é, de 5,4 a 8,1 (Carneiro, 1995), enquanto para o dedaleiro as doses 0 e $64 \mathrm{mg} \mathrm{Cu} \mathrm{kg}^{-1}$ de solo proporcionaram valores da relação H/D superiores aos da faixa adequada. Desta forma, as doses de cobre 128 e $192 \mathrm{mg} \mathrm{kg}^{-1}$ de solo ainda proporcionaram relação H/D adequada para as mudas de dedaleiro. Verifica-se, na Tabela 2, que as mudas de dedaleiro foram significativamente superiores às de timbó, na relação H/D.

As mudas de timbó apresentaram redução significativa no IQD com a aplicação das doses de cobre (Tabela 2), resultando em valores inferiores aos do índice mínimo, correspondente a 0,2, preconizado por Dickson et al. (1960), Cruz et al. (2004) e Melo et al. (2008), como indicador de alta qualidade de mudas para transplantio. Para o dedaleiro não há diferença no IQD até a dose de $128 \mathrm{mg} \mathrm{Cu} \mathrm{kg}^{-1}$ de solo; entretanto, somente nessa dose o IQD está acima do índice mínimo, sendo reduzido significativamente com $192 \mathrm{mg} \mathrm{Cu} \mathrm{kg}^{-1}$ de solo. Rossi et al. (2008) também observaram que o incremento nas doses de sulfato de cobre ocasionou redução na massa seca total do sistema radicular e no índice de qualidade de Dickson de mudas de pinus cujos resultados indicam que o efeito positivo da dose de $128 \mathrm{mg} \mathrm{Cu} \mathrm{kg}^{-1}$ de solo sob o sistema radicular das mudas de dedaleiro (Tabela 1) proporcionou melhor qualidade de mudas, expressa pelo IQD (Tabela 2), já que o IQD é calculado com base em atributos morfológicos, como a massa seca da parte aérea e do sistema radicular, que são dependentes do desenvolvimento das raízes. Esta melhora no IQD das mudas do dedaleiro com $128 \mathrm{mg} \mathrm{Cu} \mathrm{kg}^{-1}$ de solo ocasionou diferença significativa em relação ao IQD das mudas de timbó (Tabela 1).

Os resultados indicam tendência de redução linear na altura para as mudas de dedaleiro enquanto o timbó não foi afetado pelas doses testadas (Figura 2A). Para o peso da matéria seca da parte aérea o dedaleiro apresentou ponto de máxima em 47,5 mg $\mathrm{Cu} \mathrm{kg}^{-1}$ de solo enquanto o timbó apresentou redução linear (Figura 2B). Segundo Dechen \& Nachtigall (2006), a contaminação por metais pode ocasionar necrose nas folhas, desfolhamento precoce e diminuição do crescimento da planta. Neste caso, referidos 
A.

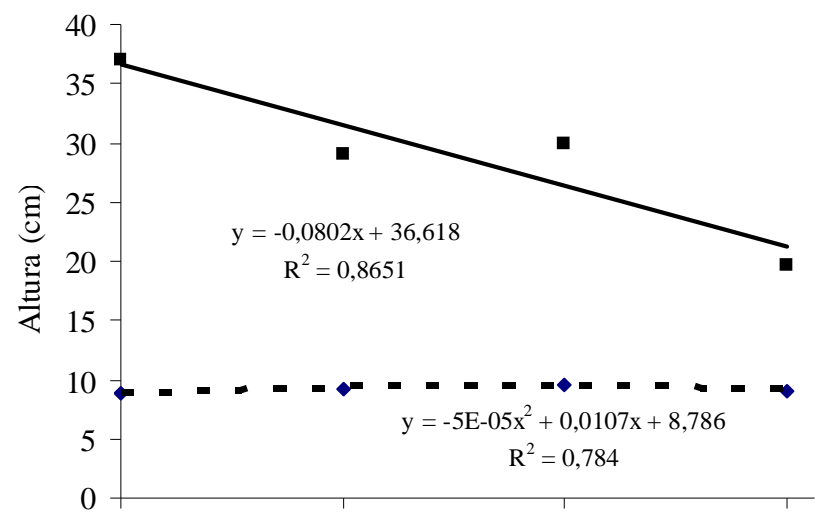

C.

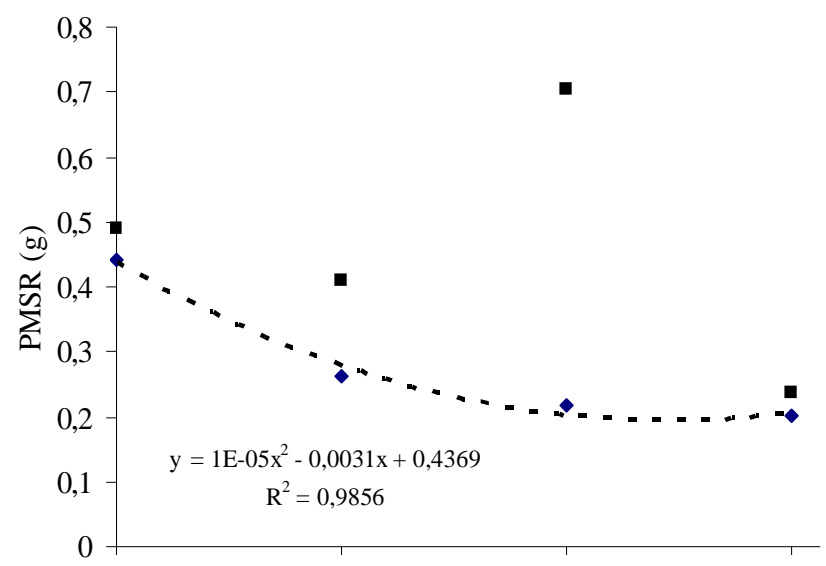

E.

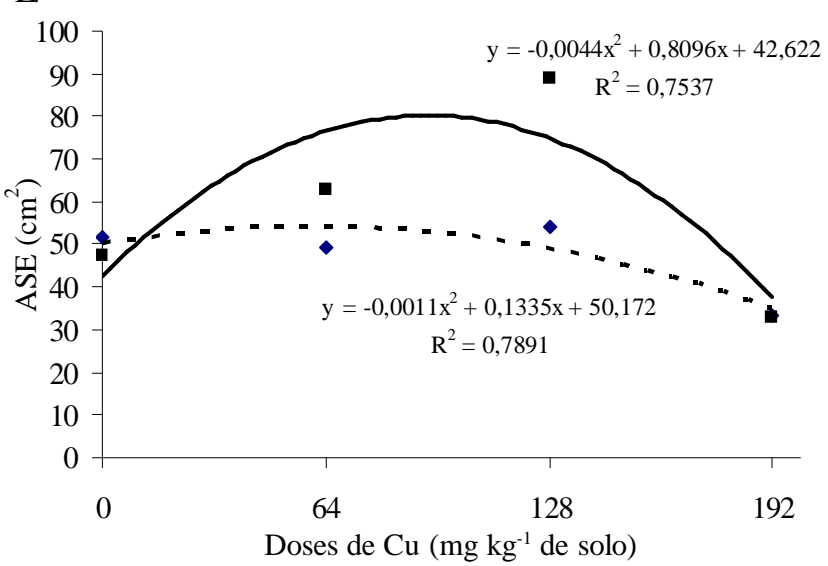

B.

- Dedalheiro

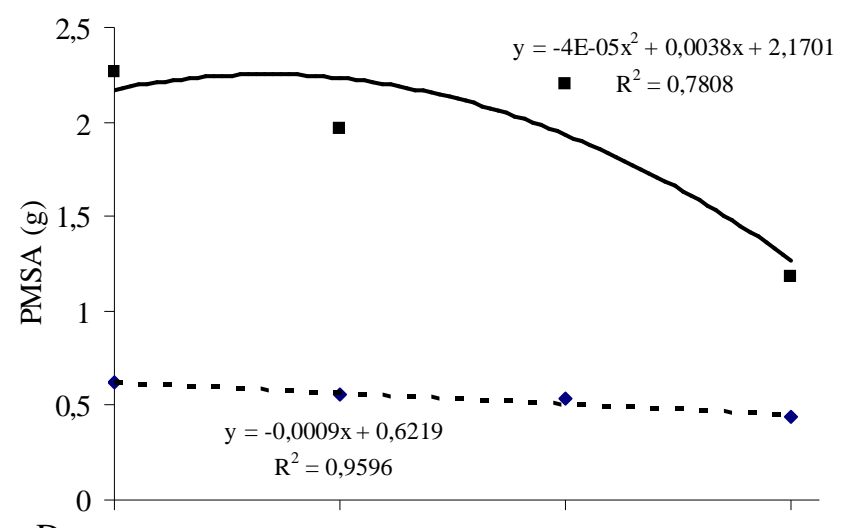

D.

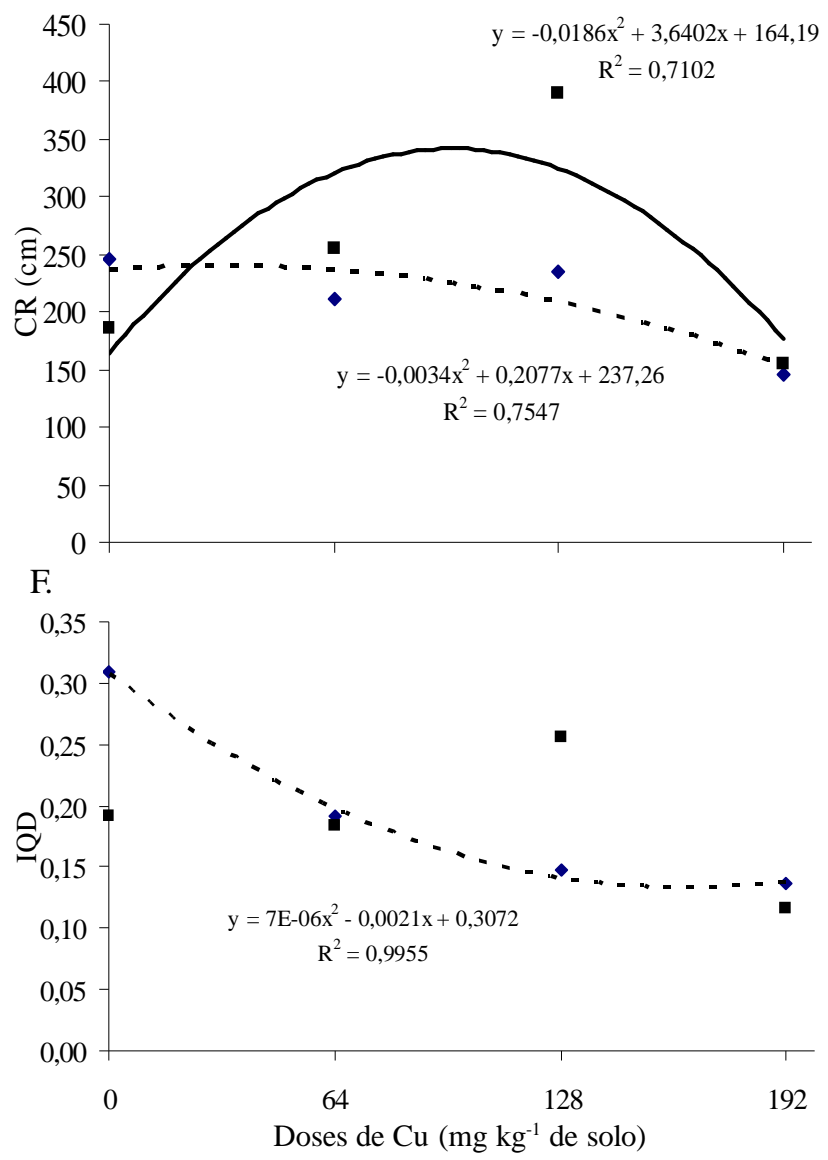

Figura 2. Altura (A), peso da matéria seca da parte aérea - MSPA (B), peso da matéria seca radicular - MSR (C), comprimento radicular - CR (D), área superficial específica radicular - ASE (E) e índice de qualidade de Dickson - IQD (F) de mudas de timbó e dedaleiro submetidas a doses de cobre

sintomas estão diretamente relacionados à redução das raízes induzida pelo cobre, que diminui a capacidade de absorção de água e nutrientes (Panou-Filotheu et al., 2001).

O dedaleiro não só revelou ponto de máxima em 97,85 $\mathrm{mg} \mathrm{Cu} \mathrm{kg}{ }^{-1}$ de solo para o comprimento radicular (Figura 2D) e $92 \mathrm{mg} \mathrm{Cu} \mathrm{kg}^{-1}$ de solo para a área superficial específica (Figura 2E) mas também evidenciou, para o peso da matéria seca da raiz, a equação $y=-2 \mathrm{e}\left(-0,5 \mathrm{x}^{2}\right)+0,0038 \mathrm{x}+0,4322 \mathrm{e}$, para o índice de qualidade de Dickson $y=-8 \mathrm{e}\left(-0,6 \mathrm{x}^{2}\right)+0,0013 \mathrm{x}+0,1768$, ambos os parâmetros com tendência de ponto de máxima porém com $\mathrm{R}^{2}$ igual a 0,43 e 0,56 , respectivamente. O timbó sinalizou redução no peso da matéria seca da raiz até $160 \mathrm{mg} \mathrm{Cu} \mathrm{kg}^{-1} \mathrm{de}$

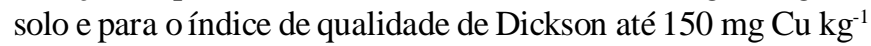
de solo; já o comprimento e a área superficial específica radicular apresentaram ponto de máxima em 30,54 e 60,68 $\mathrm{mg} \mathrm{Cu} \mathrm{kg}^{-1} \mathrm{de}$ solo, respectivamente. Conforme Bellion et al. (2006) quando o cobre é absorvido pelas raízes, provoca alteração no tecido radicular, interferindo na expansão do sistema radicular e na absorção de nutrientes. Isto ocorre pelo acúmulo de cobre nas raízes, comprometendo a permeabilidade das membranas 
(Fernández et al., 2007). Esses resultados evidenciam que o dedaleiro apresentou maior tolerância e melhor qualidade de mudas que as mudas de timbó, quando submetidas à contaminação por cobre.

\section{Conclusões}

1. As doses de cobre testadas reduzem a qualidade de mudas do timbó enquanto o dedaleiro apresenta melhor qualidade, com aplicação de $128 \mathrm{mg} \mathrm{Cu} \mathrm{kg}^{-1}$ de solo.

2. O cobre influencia os parâmetros de crescimento das mudas de timbó mas aumenta significativamente o comprimento e a área superficial específica das mudas de dedaleiro, até 128 $\mathrm{mg} \mathrm{Cu} \mathrm{kg}{ }^{-1}$ de solo.

3. Mudas de dedaleiro são mais tolerantes que as de timbó à contaminação do solo por cobre e apresentam melhor qualidade de mudas em doses mais elevadas de cobre.

\section{LITERATURA CITADA}

Accioly, A. M. A.; Siqueira, J. O. Contaminação química e biorremediação do solo. In: Novaes, R. F.; Alvarez, V. H. V.; Schaefer, C. E. G. R. (ed.) Tópicos em ciência do solo. Viçosa: SBCS, 2000. p.299-352.

Almeida, S. L.; Maia, N.; Ortega, A. R.; Ângelo, A. C. Crescimento de mudas de Jacaranda puberuba Cham. Em viveiro submetidas a diferentes níveis de luminosidade. Ciência Florestal, v.15, p.323-329, 2005.

Backes, P.; Irgang, B. Árvores do Sul - Guia de Identificação e Interesse Ecológico, 2.ed. Porto Alegre: Pallotti - Instituto Souza Cruz, 2002. 326p.

Bellion, M; Courbot, M.; Jacob, C.; Blaudez, D.; Chalot, M. Extracellular and celullar mechanisms sustaining metal tolerance in ectomycorrizal fungi. FEMS Microbiology Letters, v.254, p.173-181, 2006.

Brissette, J. C; Barnett, T. D. Container Seedlings. In: Duryea, M. L.; Dougherty, P. M. (ed.) Forest regeneration manual. Dordrecht: Kluwer Academic Publishers, 1991. p.117-141.

Carneiro, J. G. A. Produção e controle de qualidade de mudas florestais. Curitiba: UFPR/FUPEF, 1995. 451p.

Chaignon, V.; Hinsinger, P. A. Biotest for evaluating for bioavailability to plants in a contaminated soil. Journal of Environment Quality, v.32, p.834-833, 2003.

Cruz, C. A. F.; Paiva, H. N. de; Gomes, K. C. O.; Guerrero, C. R. A. Efeito de diferentes níveis de saturação por bases no desenvolvimento e qualidade de mudas de ipê-roxo (Tebebuia impetiginosa (Mart.) Standley). Scientia Forestalis, v.2, p.100-107, 2004.

Dechen, A. R.; Nachtigall, G. R. Micronutrientes. In: Fernandes, M. S. (ed.) Nutrição mineral de plantas. Viçosa: Sociedade Brasileira de Ciência do Solo. 2006. p.328-352.

Dickson, A.; Leaf, A. L.; Hosner, J. F. Quality appraisal of white spruce and white pine seedling stock in nurseries. Forest Chronicle, v.36, p.10-13, 1960.
EMBRAPA - Empresa Brasileira de Pesquisa Agropecuária Sistema brasileiro de classificação de solos. Rio de Janeiro: EMBRAPA, 2006. 412p.

Fernández, M.; Tejero, J. R.; Pérez, I.; Soria, F.; Ruiz, F.; López, G. Efeito do revestimento em cobre de contentores de viveiro sobre o crescimento e morfologia radicular de plantas provenientes de estacas de sementes de Eucalyptus globulus Labill. Silva Lusitana, v.15, p.215-227, 2007.

Ferreira, D. F. Sistemas de análise estatística para dados balanceados. Lavras: UFLA/DEX/SISVAR, 2006. 145p.

Gomes, J. M.; Couto, L.; Leite, H. G.; Xavier, A.; Garcia, S. L. R. Crescimento de mudas de Eucalyptus grandis em diferentes tamanhos de tubetes e fertilização N-P-K. Revista Árvore, v.23, p.113-127, 2003.

Marques, T. C. L. L. S. M,; Moreira, F. M. S.; Siqueira, J. O. Crescimento e teores de metais em mudas de espécies arbóreas tropicais em solo contaminado com metais pesados. Pesquisa Agropecuária Brasileira, v.35, p.121-132, 2000.

Melo, R. R.; Cunha, M. C. L.; Rodolfo Júnior, F.; Stangerlin, D. M. Crescimento inicial de mudas de Enterolobium contortisiliquum (Vell.) Morong. sob diferentes níveis de Luminosidade. Revista Brasileira de Ciências Agrárias, v.3, p.138-144, 2008.

Nachtigall, G. R.; Nogueirol, R. C.; Alleoni, L. R. F.; Cambri, M. A. Copper concentration of vineyard soils as a function of $\mathrm{pH}$ variation and addition of poultry litter. Brazilian Archives of Biology and Technology, v.50, p.941-948, 2007.

Panou-Filotheu, H. Bosabalidis, A. M.; Karataglis, S. Effectes of copper toxicity on leaves of oregano (Origanum vulgare subsp. Hirtum). Annals of Botany, v.88, p.207-214, 2001.

Pralon, A. Z.; Martins, M. A. Utilização do resíduo industrial Ferkal na produção de mudas de Mimosa caelsalpinifolia, em estéril de extração de argila, inoculadas com fungos micorrízicas arbusculares e rizóbio. Revista Brasileira de Ciência do Solo, v.25, p.55-63, 2001.

Rossi, V. L.; Amarante, C. V. T.; Fleig, F. D. Crescimento e qualidade de mudas de Pinus taeda L. submetidas à poda química de raízes. Ciência Florestal, v.18, p.435-442, 2008.

Souza, C. A. M.; Oliveira, R. B.; Martins Filho, S.; Lima, J. S. S. Crescimento em campo de espécies florestais em diferentes condições de adubação. Ciência Florestal, v.16, p.243-249, 2006.

Souza, V. C.; Andrade, L. A.; Bruno, R. L. A; Cunha, A. O; Souza, A. P. Produção de mudas de ipê-amarelo (Tabebuia serratifolia (Vahl.) Nich.) em diferentes substratos e tamanhos de recipientes. Agropecuária Técnica, v.26, p.98$108,2005$.

Tennant, D. A test of a modified liwe intersect method of estimating root lengh. Journal Ecology, v.63, p.995-1001, 1975.

Ter-Braak, C. J. F.; Smilaeur, P. CANOCO reference manual and user's guide to Canoco for Windows: Software for canonical community ordination (version 4). New York: Microcomputer Power, 1998. 